\title{
A sounding rocket experiment to control boiling by means of acoustic waves
}

\author{
G. Quintana-Buil · A. Garcia-Sabaté · \\ S. Batlle · G. López • V. Sierra • R. \\ González-Cinca
}

Received: date / Accepted: date

\begin{abstract}
One of the most critical issues when considering long-term space exploration missions is the management and storage of cryogenic propellants. The exposure of storage tanks to radiation and extreme temperatures implies the need of efficient technologies to counteract their effects on the fuel. A potentially dangerous effect for spacecraft operations is the generation of vapor bubbles in cryogenic propellants. We present an experimental setup and procedure to mature a technology based on acoustic waves to control boiling in microgravity.
\end{abstract}

Keywords Boiling · Acoustics and Microgravity

\section{Introduction}

Future long-term space exploration missions will require efficient fuel storage in microgravity. Cryogenic propellants (affordable cost, environmentally friendly) are good candidates for these missions. However, their storage conditions can be very demanding, particularly regarding temperature. Liquid hydrogen, for example, is stored at $20 \mathrm{~K}$. In long-term missions, the persistent solar incidence on the fuel tanks can cause heat leaks which in turn could generate localized boiling leading to bubble formation. Vapor bubbles under reduced g-forces cannot rise up the liquid phase as in terrestrial conditions and its accumulation can be hazardous for several vehicle manouvres such as engine restart or propellant loading [1].

The analysis of the characteristics of boiling in microgravity was first addressed by Siegel and Keshock [2], and it has been followed by many other authors $[3,4]$. The use of acoustic waves is an efficient method to manage the dynamics of gas or vapor bubbles. Bubbles in a standing acoustic wave are

G. Quintana-Buil, A. Garcia-Sabaté, S. Batlle, G. López, V. Sierra, and R. González-Cinca Department of Physics, Universitat Politècnica de Catalunya-BarcelonaTech, c/ E. Terradas 5, 08860 Castelldefels, Barcelona, Spain 
driven to pressure nodes or antinodes depending on their size [5]. Acoustic fields can also modify heat transfer in boiling on ground [6-8] and in microgravity [9].

In this paper we present an experimental setup and procedure to study bubble dynamics in pool boiling under the application of an acoustic field in microgravity. The setup was selected by the European Space Agency's Education Office to fly in a sounding rocket under the framework of the REXUS/BEXUS programme.

\section{Experimental setup}

The experimental setup was designed taking into account the characteristics of the REXUS vehicle (specifications, interfaces, microgravity conditions) and with the aim of acquiring valuable information of the phenomenon of study. The setup (Fig. 1) consists of a test cell and systems for boiling generation, acoustic field generation, data acquisition, and experiment control. A cylindrical module (height $220 \mathrm{~mm}$, diameter $356 \mathrm{~mm}$ ) contains the setup in two levels. The test cell and most parts of the systems and electronics are in the upper level. The computer is the only equipment in the lower level. Fig. 2 shows a block diagram with the internal connections in the experimental setup.

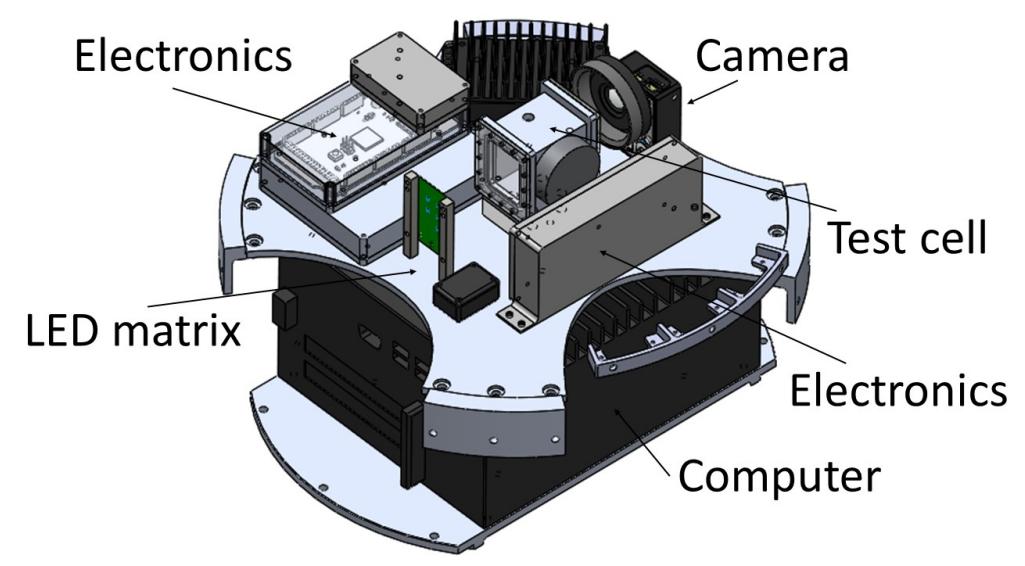

Fig. 1 Experimental setup.

When the rocket reaches the microgravity phase, bubbles are formed in the test cell by activating a heater in it, while an acoustic field is generated to act on the bubbles. The experiment is monitored by different sensors. The bubble dynamics is recorded by means of a video camera. 


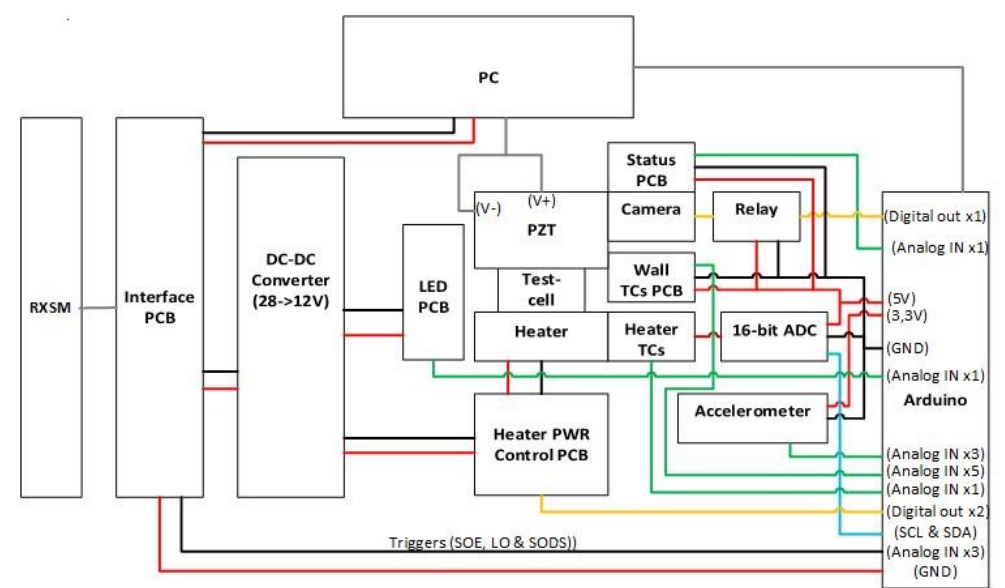

Fig. 2 Block diagram of the connections in the experimental setup. Red: power, black: ground, green: analog signal, blue: digital signal, yellow: trigger signal.

\subsection{Test cell}

48 The physical phenomenon of study takes place in the test cell (Fig. 3). Several 49 tests were carried out to determine the optimal final design for the purpose of 50 the experiment.

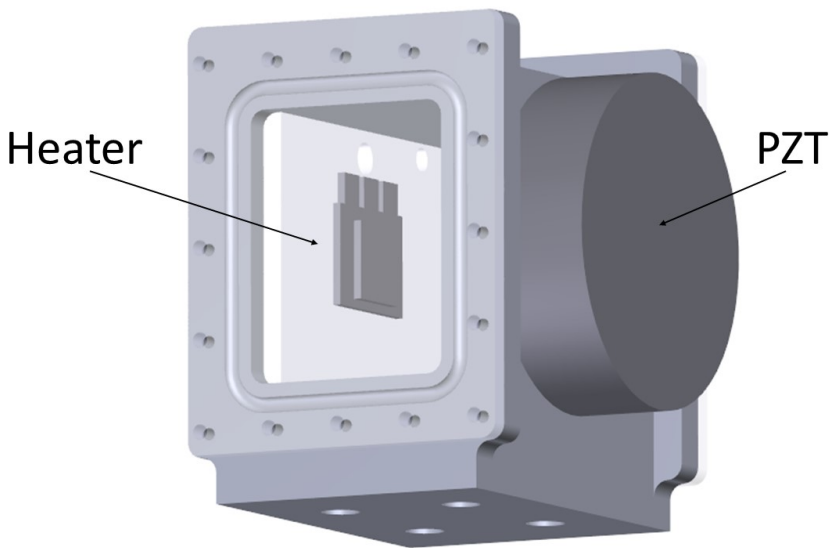

Fig. 3 Test cell.

The cell is made of Aluminum T6061 with dimensions (HxWxL) 61 x $45 \mathrm{x}$ $58 \mathrm{~mm}$ containing HFE7100. The material was chosen given its good structural properties for the hypergravity conditions at rocket launch, and given the appropriate acoustic properties for the propagation of the wave generated in a piezoelectric ceramic (PZT). In order to provide visual access to the inside 
of the cell, it has two windows made of transparent PMMA. The PZT is attached by conductive epoxy to the outside of one of the walls perpendicular to the windows. On the opposite wall, a heater is placed to heat the liquid and generate boiling. The test cell is connected to a waste tank through an overpressure valve, so that pressure is kept constant inside the cell and boiling temperature does not change during the experiment. The connection is used before the experiment to fill the cell by means of a syringe and a second valve.

\subsection{Boiling generation}

The objective of the boiling generation system is to heat the liquid in the cell in a controlled way so that bubbles are generated at an inner wall of the test cell at an appropriate rate for the purpose of the experiment. Bubbles are generated by means of a $1 \mathrm{~cm}^{2}$ area and $0.42 \mathrm{~mm}$ thickness Captec (www.captec.fr) heating element (Fig. 4), which consists of an electrical resistance heated by Joule effect in contact with a heat flux meter and a copper plate. The same type of heater has been used in pool boiling experiments in microgravity [10]. The heating resistance of $27.3 \Omega$ has a serpentine shape over the surface. Heat flux transmitted to the liquid and temperature can be measured by means of the flux meter and two thermocouples. Measurements are controlled and recorded by an Arduino hardware platform used as an acquisition data module. The copper layer (30 $\mu \mathrm{m}$ thickness) is in contact with the fluid and separated from the flux meter by a polyamide layer of thickness $150 \mu \mathrm{m}$.
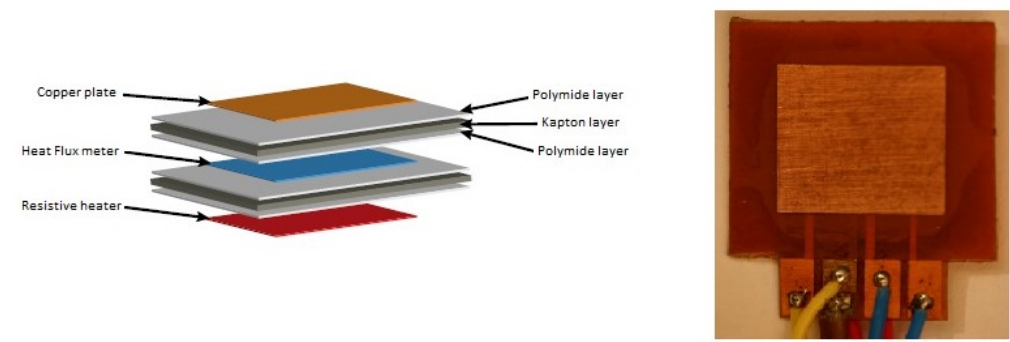

Fig. 4 Heating element. Left: side view, right: top view (www.captec.fr).

The heating power is supplied by a DC-DC converter which offers a maximum voltage of $12 \mathrm{~V}$. The applied voltage (either $6 \mathrm{~V}$ or $12 \mathrm{~V}$ ) is controlled by an Arduino Mega 2560 board.

\subsection{Acoustic field generation}

The objective of the acoustic wave generation system is to create an acoustic field in the liquid that can modify the dynamics of the bubbles. For this pur- 
pose, a standing wave is generated inside the test cell by means of an electrical signal applied to the PZT. The system is composed of the following elements:

- Function generator (Tabor Electronics 5325 PCI card) to generate and control the frequency and voltage amplitude of the applied sinusoidal wave.

- Amplifier (10x voltage amplifier Tabor Electronics 3322 PCI card). The low output voltage amplitude from the function generator leads to a low acoustic energy in the liquid, which is insufficient to alter the bubble dynamics. This makes necessary the use of the amplifier, which can provide up to $40 \mathrm{Vp}-\mathrm{p}$.

- PZT with transverse nominal frequency of $160 \mathrm{kHz}$. It is connected to the amplifier output and converts the electrical signal into a mechanical vibration.

- Computer (MXC 4000/2G, AdLink Technology Inc.). It contains the function generator and amplifier PCI cards, supplying power to them and controlling them by means of a LabView code. The computer has no moving parts (no fan, SDD).

A sinusoidal standing wave is generated between the wall with the PZT and the opposite wall where the heater is attached. The frequency of operation is selected under several criteria. The frequency must generate a pressure node at the heater. Since bubbles are smaller than the resonance size, they are attracted to the pressure antinodes, thus contributing to the detachment of the bubbles from the heater. Moreover, the working frequency is selected so that there is a small number of nodes (between 3 and 5) in the liquid. This ensures a long enough bubble path towards the antinodes. In addition, resonance frequencies or their harmonics are preferred since they provide larger vibration amplitudes and consequently a larger acoustic force on the bubbles.

Several tests were carried out to determine the appropriate frequency of the acoustic standing wave according to the above criteria. The amplitude of the wave in the liquid was measured by means of an hydrophone. Fig. 5 shows the acoustic pressure of a wave of $39 \mathrm{kHz}$ in the axis crossing the PZT and heater walls at their center. The PZT and the heater are at $\mathrm{x}=0 \mathrm{~mm}$ and $\mathrm{x}=27 \mathrm{~mm}$, respectively. The experimental line corresponds to the measurements obtained with the hydrophone. The theoretical line corresponds to the expected form of the wave.

\subsection{Data acquisition}

Two types of data are recorded during the experiment for later analysis. A GoPro Hero 3+ Black Edition video camera (up to 1280x720 pixels at 120 fps) records the phenomenology taking place inside the test cell. The computer triggers the camera and data is stored in an SD card. The required light is provided by a matrix of LEDs. A diffuser sheet provides homogeneous background illumination. 


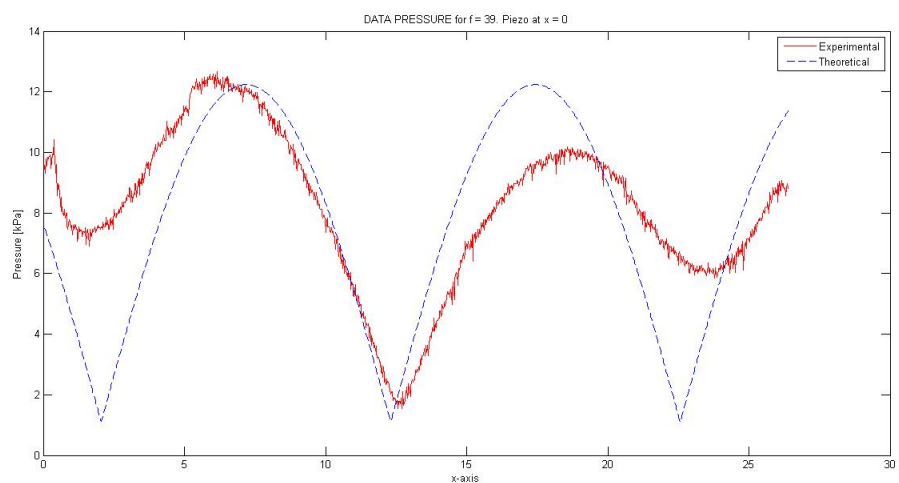

Fig. 5 Acoustic pressure in the axis perpendicular to the PZT and heater walls. Th PZT and the heater are at $\mathrm{x}=0 \mathrm{~mm}$ and $\mathrm{x}=27 \mathrm{~mm}$, respectively.

An accelerometer and a set of thermocouples also provide data on the experiment. The Arduino board is used as a data acquisition device for these sensors. Since Arduino has only 10 bits of resolution, a 16 bit ADC (ADS1115 from Adafruit) is placed between the heater thermocouples and flux meter and the Arduino board in order to obtain a higher resolution. One of the thermocouples is a T-type and the data acquisition is made by means of a cold junction compensator (AD595). Data from the other two sensors are acquired using a 16-bit ADC (ADS1115). The second thermocouple has a sensitivity of $32 \mu \mathrm{V} / \mathrm{C}$ and is compensated by the first one.

A set of five thermocouples type $\mathrm{T}$ are distributed inside the test cell to obtain information on the temperature of the liquid, and at different locations of the experimental setup to measure the temperature of other devices. The Arduino board acquires the temperature measurements from the sensors through a cold junction compensator (AD595) that linearizes the ouput signal. The signal is amplified and filtered in order to achieve the desired resolution and to reduce the noise.

The accelerometer is placed on the longitudinal axis of the rocket in order to record the g-jitter and peaks of acceleration that could affect the bubble dynamics.

\subsection{Control software}

A LabView 2013 (National Instruments) code was built to control the following processes:

- Boiling generation (heater power supply).

- Acoustic wave generation (function generation and amplifier).

- Thermocouples and accelerometer data acquisition and storage.

- Uplink and downlink. 
The code includes two optional working modes: Nominal and Test. The Nominal mode is planned for the actual flight. The Test mode is used for tests with the setup in the lab or in the rocket in which the boiling system must not be activated. In this mode, the experiment trasmits to the ground segment the correct reception of the trigger signals, but does not activate their corresponding protocols. In addition, no data is stored in the Test mode. The code allows 5 minutes for the mode selection. If no signal has been received after this time, the nominal mode is launched.

The control software is divided in several stages, most of them running simultaneously. At first, the main variables are initialized and the status values are set to zero. In addition, the Arduino and the function generator are initialized and the output file headers are created. It continues with the temperature and acceleration measurements acquisition from the Arduino analogical inputs. At some point the main protocols for boiling and acoustic wave generation, and camera switch on are activated. The computer is switched off after all the protocols have finished.

The software allows the experiment to be controlled by telemetry. The following uplink actions are available from the ground segment:

- To start the experiment.

- To check if uplink is working.

- To select a mode (Test or Nominal).

- To stop Test mode (in order to run Nominal mode).

- To shutdown the experiment.

- To switch on/off the camera.

The ground segment is able to receive all the experimental data except from the video.

\section{Experimental procedure}

At computer switch on (10 minutes before lift-off), the LabView code is launched and waits for the first uplink signal to select between the Test and the Nominal mode. In case no signal is received, the Nominal mode starts 5 minutes before the lift-off signal. The start and end times of boiling and acoustic wave generation systems are fixed according to the specific rocket flight characteristics (time of motor separation). Since the estimated time for the microgravity phase is 134 seconds, the camera and the computer are switched off 5 and 10 minutes after lift-off, respectively.

The aim of the experimental protocol is to manage bubble dynamics by detaching and moving them away from the heater. The acoustic wave amplitude is kept constant during the experiment. A first set of three acoustic wave frequencies around $35 \mathrm{kHz}$ is applied for 15 seconds each with the heater on just after microgravity conditions are reached. Since this phase starts immediately after the rocket de-spin and booster separation, the bubble dynamics can be affected by the g-jitter. The experiment continues with the application 
of a pair of high $(168 \mathrm{kHz})$ and low $(35 \mathrm{kHz})$ frequencies. The high frequency, which is closer to the PZT resonance frequency, is applied for 45 seconds with the aim at detaching and moving the bubbles. The low frequency is applied for 9 seconds to move the bubbles to the corresponding antinodes. In the last 35 seconds of the microgravity phase, the pair of high and low frequencies are applied in the same way with a larger power applied to the heater in order to generate more bubbles.

All frequencies are applied in sets of three ( $\mathrm{f}, \mathrm{f}+\Delta f$ and $\mathrm{f}-\Delta f$ ) to allow slight displacements of the position of the nodes and antinodes of the generated wave.

\section{Conclusions}

We have presented an experimental setup and the corresponding procedure to study the dynamics of bubbles generated by boiling under the application of an acoustic field in microgravity. The design of the setup is determined by the scientific objectives and the characteristics of the sounding rocket. The experimental setup run successfully in the REXUS rocket launched in spring 2016. The analysis of the obtained data will be published elsewhere [11]. The scaling of the setup for a further maturation of the acoustic technology in other microgravity platforms is straightforward.

Acknowledgements This research was supported by the Spanish Ministerio de Economía y Competitividad, Secretaría de Estado de Investigación, Desarrollo e Innovación (project number AYA2012-34131), and by the Agencia Estatal de Investigación and EU FEDER (project number ESP2016-79196-P). Authors acknowledge ESA Education Office for providing access to the REXUS/BEXUS programme, and ESA, ZARM, and SNSB for technical assistance. We thank M. Khasin and C. Muratov for fruitful discussions.

\section{References}

1. C. B. Muratov, V. V. Osipov, and V. N. Smelyanskiy, Issues of long-term cryogenic propellant storage in microgravity, NASA/TM-2011-215988 (2011).

2. R. Siegel, E. G. Keshock, Effects of reduced gravity on nucleate boiling bubble dynamics in saturated water, AIChE Journal 10, 509 (1964).

3. J. Straub, M. Zell, B. Vogel, What we learn from boiling under microgravity, Microgravity Sci. Technol. 6, 239 (1993).

4. P. Di Marco, Review of reduced gravity boiling heat transfer: European research, J. Jpn. Soc. Microgravity Appl. 20, 252 (2003).

5. T. G. Leighton, A. J. Walton, M. J. W. Pickworth, Primary Bjerknes forces, Eur. J. of Physics 11, 47 (1990).

6. S. E. Isakoff, Effect of an ultrasonic field on boiling heat transfer - Exploratory investigation, Heat Transfer and Fluid Mechanics Institute, Stanford University Press (1956).

7. Z. W. Douglas, T. R. Boziuk, M. K. Smith, and A. Glezer, Acoustically enhanced boiling heat transfer, Phys. of Fluids 24, 052105 (2012).

8. S. Krishnan, S. K. Das, D. Chatterjee, Physics of the interaction of ultrasonic excitation with nucleate boiling, J. Heat Transfer 136, 031501 (2014).

9. J. S. Sitter, T. J. Snyder, J. N. Chung, and P. L. Marston, Terrestrial and microgravity pool boiling heat transfer from a wire in an acoustic field, International Journal of Heat and Mass Transfer 41, 2143 (1998). 
236 10. O. Kannengieser, C. Colin, W. Bergez, Pool boiling with non-condensable gas in mi237 crogravity: results of a sounding rocket experiment, Microgravity Sci. Technol. 22, 447 $238 \quad(2010)$

239 11. G. López and R. González-Cinca (in preparation). 